\title{
Riqueza, distribución y estado de conservación de las especies de encino en Guatemala
}

\author{
Richness, distribution, and conservation status of the species of oak in Guatemala \\ Maura Liseth Quezada*, Rosario Rodas-Duarte, Daunno Chew, Andrea A. Marroquín-Tintí \\ Centro de Estudios Conservacionistas (Cecon), Facultad de Ciencias Químicas y Farmacia, \\ Universidad de San Carlos de Guatemala, Guatemala
}

*Autor al que se dirige la correspondencia: herbariouscg@usac.edu.gt

Recibido: 23 de febrero 2017 / Revisión: 09 de octubre 2017 / Aceptado: 23 de noviembre 2017

\section{Resumen}

as especies del género Quercus son componentes estructurales de los bosques templados y semicálidos en
Guatemala, así como la principal fuente energética en las zonas rurales del país. A pesar de su importancia
aún se desconoce la riqueza, distribución y grado de vulnerabilidad de las especies. Por lo cual, se presenta una
actualización del listado de especies para Guatemala, su distribución potencial y la evaluación del estado de
conservación con base a los criterios utilizados para el listado nacional de especies amenazadas (LEA). Se re-
gistraron 29 especies de encinos, 12 corresponden a encinos blancos (Sect. Quercus) y 17 a encinos rojos (Sect.
Lobatae), de los cuales $Q$. cortesii, $Q$. gulielmi-treleasei, $Q$. vicentensis, Q. xalapensis se citan por primera vez
para Guatemala. Las especies con mayor número de registros son $Q$. sapotifolia (13\%), $Q$. peduncularis (13\%),
Q. crassifolia (12\%), Q. skinneri (7\%) y Q. acutifolia (6.5\%), correspondiendo a más del $50 \%$ de los registros.
Los departamentos con mayor número de registros son Guatemala (177), Zacapa (155), Huehuetenango (134),
Baja Verapaz (115) y Chiquimula (93); mientras que departamentos como Retalhuleu, Escuintla y Suchitepéquez
presentan menos de 10 registros. En relación al estado de conservación, seis se ubicaron En Peligro Crítico, 22 en
la categoría Amenazada y una en la categoría Vulnerable.

Palabras claves: Quercus, robles, sitios prioritarios, distribución, bosques energéticos.

Abstract

$\mathrm{T}$

he species of the genus Quercus, commonly known as oaks, are important structural components of the forests in the region, as well as a primary source of energy in the rural areas of Guatemala. Despite their relevance, the richness, distribution and degree of vulnerability of the species, are still unknowing. Therefore, it presented an update of the list of species for Guatemala, its potential distribution and the assessment of the state of conservation based on the criteria used for the List of Threatened Species (LEA in Spanish). Twenty-nine species of oak were recorded, of which $Q$. cortesii, Q. gulielmi-treleasei, Q.vicentensis and $Q$. xalapensis are cited for the first time for Guatemala. The species with most number of records are $Q$. sapotifolia $(13 \%), Q$. peduncularis $(13 \%)$, Q. crassifolia (12\%), Q. skinneri. (7\%) and Q. acutifolia (6.5\%), that represent more than $50 \%$ of the records. The departments with the highest number of records are Guatemala (183), Zacapa (155), Huehuetenango (134) and Baja Verapaz (115), while departments such as Retalhuleu, Escuintla and Suchitepéquez present less than 10 records. Regarding the state of conservation, the species were evaluated using the methodology of the List of Threats Species of Guatemala, six species were Critically Endangered, 22 in the Threatened category and one in the Vulnerable category.

Keywords: Quercus spp., oaks, research priorities, potential distribution, energy species. 


\section{Introducción}

El género Quercus posee la mayor riqueza de especies y es el más ampliamente distribuido a nivel mundial dentro de la familia Fagaceae, encontrándose en casi todos los bosques templados del hemisferio norte, y en algunas regiones tropicales y subtropicales (Manos, Doyle y Nixon, 1999; Nixon, 2006; Valencia-A, 2004). Se estima que hay más de 500 especies en el mundo y para Centroamérica se reconocen alrededor de 40 especies (Manos et al., 1999; Muller, 1942; Nixon, 2006). Los encinos o robles (Quercus sp.) constituyen un componente importante de la estructura de los bosques neotropicales, están asociados a diferentes ecosistemas como bosques secos y deciduos, bosques montanos, selvas lluviosas, entre otros. Estas especies pueden encontrarse a nivel del mar hasta altitudes arriba de los 3,000 m (Kappelle, 2006; Nixon, 2006; Pérez-Ramos, Villar, \& Marañon, 2014; Rzedowski, 1978; Valencia-A., 2004; Zavala-Chavez, 1998).

Muller (1942) cita para Guatemala 24 especies de encinos y dos subespecies; mientras que Standley y Steyermark (1952), citan 26 especies y dos subespecies para el país. Estas especies se clasifican en dos secciones propuestas por Nixon (2006): sección Lobatae (encinos rojos) y sección Quercus s.s (encinos blancos). Sin embargo, éstas especies son poco conocidas posiblemente por su taxonomía complicada, dado que la determinación se realiza utilizando caracteres foliares. Estas especies presentan una alta variación inter e intra específica, así como una alta hibridación, presentando características intermedias entre las formas parentales (Torres-Miranda, 2014), lo que dificulta su identificación, además que se conoce poco sobre aspectos particulares de su distribución como el tipo de hábitat, suelo, clima, entre otros (Zavala-Chavez, 1998).

En 2015 se inició la actualización sistemática de este grupo en el nororiente de país (Quezada, Rodas-Duarte, \& Marroquín-Tintí, 2016; 2016b) considerando su importancia ecológica y socioeconómica. Ecológicamente, las especies de éste género son consideradas clave en la estructura de los bosques, dada la provisión de hábitat y alimento para otras especies. Asimismo, contribuyen en los procesos de regulación como la filtración de agua, secuestro de carbono, formación y protección del suelo, entre otros (Maranón, Padilla-Díaz, Pérez-Ramos, \& Villar, 2014; Moreno, 2006). Socialmente, este género se registra como el más utilizado como fuente energética por el $70 \%$ de la población rural, la cual aún depende de la leña para la cocción de alimentos y calefacción (Melgar, 2003). Del mismo modo, la mayoría de hongos comestibles están asociados a estos bosques (Flores, Comandini, \& Rinaldi, 2012), además los bosques asociados a estas especies son utilizados para realizar actividades recreativas como caminatas, aviturismo, ciclismo de montaña, entre otras.

Dada la importancia económica de este grupo, el Consejo Nacional de Áreas Protegidas (Conap) cita que más de $80 \%$ de las especies se encuentran dentro del Listado Nacional de Especies Amenazadas (LEA). La poca información en cuanto a su riqueza y distribución, así como la pérdida de hábitat, cambio de uso y la sobreutilización del recurso, hacen que sea un grupo muy vulnerable (Alianza para la Conservación de Bosques Pino-Encino, 2010; Conap, 2009). Por lo anterior, el objetivo de este estudio es aportar a la actualización de la riqueza y distribución de las especies de Quercus, el estado de conservación y los vacíos de colectas del género para el país. Se espera que esta información sirva como base para la priorización de especies y áreas geográficas, así como para elaborar estrategias de manejo, regulación y conservación del género que permitan la permanencia de las especies en el tiempo y con ello los bienes y servicios que proporcionan a la sociedad guatemalteca.

\section{Materiales y métodos}

\section{Recolección de datos}

Los datos de los registros de las especies fueron recabados de varias fuentes que se detallan a continuación:

Revisión de literatura. Se revisaron publicaciones del género Quercus como floras, lista de especies, artículos, documentos técnicos y tesis de grado. Estos escritos proporcionaron datos de número de especies por departamento, sinonimias, datos de distribución, localidades, estado de conservación y uso tradicional de las especies (Balick, Nee, \& Atha, 2000; Conap, 2009; García, 1998; Muller, 1942; Quezada et al., 2016, 2016b, Rodríguez-Correa, Oyama, MacGregor-Fors, \& González-Rodríguez, 2015; Standley \& Steyermark, 1952; Stevens, Ulloa, Pool, \& Montiel, 2001; Torres-Miranda, 2014; Valencia-A, 2004; Valencia-A, Franco, \& Ramírez, 2015; Vivero, Szejner, Gordon, \& Magin, 2006). 
Revisión de material de herbarios nacionales. Se revisaron las colecciones de referencia del género Quercus disponibles en los herbarios nacionales: Herbario de Biología de Guatemala (Bigu), Herbario de Agronomía de Guatemala (Aguat), Herbario de la Universidad del Valle (Uval) y Herbario de la Universidad de San Carlos de Guatemala (Uscg).

Revisión de bases de datos de herbarios extranjeros. Las bases de datos consultadas fueron: Global Biodiversity Information Facility (GBIF), Tropicos ${ }^{\circledR}$ del Herbario del Missouri Botanical Garden (MO), Muestras Neotropicales de Herbario del Field Museum of Natural History (F), Herbario del Royal Botanical Garden De Kew (K), Botanischer Garten und Botanisches Museum Berlin-Dahlem, Zentraleinrichtung der Freien Universität Berlin, Alemania (B), Natural History Museum, Londres (BM), Smithsonian (US), University of Notre Dame (ND), Museo Nacional de Costa Rica (CR), University of Illinois (ILL), Universidad de California (UC). De las bases de datos consultadas se extrajeron los siguientes datos por registro: especie, localidad de la colecta, coordenadas, altitud, fecha de colecta y colectores.

\section{Procesamiento de los datos.}

Actualización de listado de encinos para Guatemala. Con los datos recabados en las colecciones de herbario nacionales y extranjeras de los registros de encinos para el país, se elaboró una base de datos en una hoja de cálculo. Esta base de datos incluye información de la especie, localidad de la colecta, coordenadas geográficas, altitud, fecha de colecta y colectores. Los registros que presentaban datos incompletos de localidad fueron georreferenciados, con base al Diccionario Geográfico Nacional (Gall, 1999). Cabe destacar que no se consideraron los registros a los que no fue posible confirmar su identidad taxonómica y aquellos cuya localidad registrada esta a nivel de municipio. La información obtenida fue revisada y depurada por medio de la verificación de sinonimias y nombres actuales utilizando el sitio web de The Plant List v. 1.1 (http:// www.theplantlist.org/), así como publicaciones recientes (Balick et al., 2000; Rodríguez-Correa et al., 2015; Torres-Miranda, 2014; Valencia-A, 2004; Valencia-A, et al, 2015); obteniendo el listado actualizado de especies para el país.

Distribución potencial de las especies de encino. Los mapas de distribución por especie se genera- ron a partir de la base de datos obtenida y modelos de temperatura, precipitación y altitud a nivel nacional, utilizando el programa ArcGIS (versión 10.3). El modelo de temperatura se obtuvo a partir de una regresión de los datos de temperatura y altitud de las estaciones meteorológicas del país de los años 1986-1996 proporcionados por el Instituto Nacional de Sismología, Vulcanología, Meteorología e Hidrología (Insivumeh). El modelo de precipitación se generó a través de una regresión lineal utilizando el mapa de isoyetas de 1996 (Ministerio de Agricultura y Alimentación [Maga] \& Instituto Geográfico Nacional [IGN], 2003). Para generar los datos de altitud se utilizó el modelo de elevación nacional (Maga \& IGN, 2006). Con base a estos tres modelos, cada registro de herbario de Quercus se correlacionó según sus coordenadas con los datos de temperatura, humedad y altitud. Para cada especie de encino se realizó una yuxtaposición de los tres modelos y se obtuvo el valor máximo y mínimo para cada una de estas variables. Con base a los valores máximos y mínimos por especie se generaron lo mapas de distribución, donde los datos de cada pixel corresponde a los datos de temperatura, precipitación y altitud dentro del rango generado para cada especie. Posteriormente se realizó un mapa con la yuxtaposición de las capas de distribución potencial para cada una de las especies de encino, obteniéndose los sitios con mayor traslape de especies.

Para complementar el mapa de distribución potencial de las especies de encino, se contrastó con la capa de cobertura 2012 (Grupo Interinstitucional de Monitoreo de Bosques y Uso de la Tierra; 2014). El mapa de cobertura 2012 se trabajó con base a un análisis booleano, donde se categorizó bosque $=1$, y otros usos como no bosque $=0$. A la capa resultante se le realizó una intersección con las capas de distribución potencial de las especies de encino y el Sistema Guatemalteco de Áreas Protegidas (Sigap) (Conap, 2016). Posteriormente se obtuvo los mapas de distribución potencial de encinos en remanentes boscosos de Guatemala, distribución potencial de encinos en remanentes boscosos fuera y dentro del Sigap. Con base a los registros de herbario, se establecieron las áreas con mayor densidad de colecta y posteriormente las áreas con vacíos de información utilizando una interpolación de Kernel de $10 \mathrm{~km}$ y luego reclasificando el resultado en imagen booleana.

Evaluación del estado de conservación. Tomando como base la información depurada para cada una de las especies, se realizó la evaluación del estado de conservación utilizando los criterios para la elabora- 
ción del listado nacional de especies amenazadas (LEA) (Fonacon, s.s). Los criterios utilizados son: (1) Estado de la población; (2) Distribución y/o endemismo; (3) Estado de hábitat y (4) Impacto de la actividad humana y del comercio sobre las especies. Para cada especie se realizó el ejercicio de evaluar cada uno de los criterios y se propone su ubicación en cada una de las categorías propuestas para el listado de especies amenazadas. Asi- mismo, se realizó una revisión de la categorización de las especies de encino según la Lista Roja de Especies Amenazadas propuesta por la Unión Internacional para la Conservación de la Naturaleza (UICN) en evaluaciones mundiales, regionales y nacionales (Jerome, 2017; Nixon, 1998; Vivero, Szejner, Gordon, \& Magin, 2006; Wenzell \& Kenny, 2015).

Tabla 1

Listado actualizado de especies del género Quercus para Guatemala

\begin{tabular}{|c|c|c|c|}
\hline No. & Sección & Nombre Aceptado & Distribución Regional \\
\hline 1 & \multirow{12}{*}{$\begin{array}{l}\text { Quercus s.s., Subge- } \\
\text { nus Lepidobalanus } \\
\text { (Encinos blancos) }\end{array}$} & Quercus bumelioides Liebm. & ME, GU, BE, ES, HO, NI, CR, PA \\
\hline 2 & & Quercus corrugata Hook. & $\mathrm{ME}, \mathrm{GU}, \mathrm{BE}, \mathrm{ES}, \mathrm{CR}$ \\
\hline 3 & & Quercus insignis M. Martens \& Galeotti & ME, GU, BE, ES, HO, NI, CR, PA \\
\hline 4 & & Quercus lancifolia Schltdl. \& Cham. & $\mathrm{ME}, \mathrm{GU}, \mathrm{HO}, \mathrm{CR}$ \\
\hline 5 & & Quercus oleoides Schltdl. \& Cham. & $\mathrm{ME}, \mathrm{GU}, \mathrm{HO}, \mathrm{NI}, \mathrm{CR}$ \\
\hline 6 & & Quercus pacayana C.H. Mull. & $\mathrm{GU}, \mathrm{HO}, \mathrm{CR}, \mathrm{PA}$ \\
\hline 7 & & Quercus peduncularis Née & $\mathrm{ME}, \mathrm{GU}, \mathrm{BE}, \mathrm{ES}, \mathrm{HO}, \mathrm{NI}$ \\
\hline 8 & & Quercus polymorpha Schltdl. \& Cham. & $\mathrm{ME}, \mathrm{GU}$ \\
\hline 9 & & Quercus purulhana Trel. & ME, GU, BE, HO, NI \\
\hline 10 & & Quercus rugosa (Masam.) J.C.Liao & $\mathrm{ME}, \mathrm{GU}$ \\
\hline 11 & & Quercus segoviensis Liebm. & $\mathrm{ME}, \mathrm{GU}, \mathrm{HO}, \mathrm{NI}$ \\
\hline 12 & & Quercus vicentensis Trel.* & ME, GU, BE, ES, HO, NI, CR, PA \\
\hline 13 & \multirow{17}{*}{$\begin{array}{c}\text { Lobatae, Subgenus } \\
\text { Erythrobalanus } \\
\text { (Encinos negros } \\
\text { o rojos) }\end{array}$} & Quercus acatenangensis Trel. & ME, GU, ES, HO \\
\hline 14 & & Quercus acutifolia Née & $\mathrm{ME}, \mathrm{GU}$ \\
\hline 15 & & Quercus benthamii A. DC. & ME, GU, ES, HO, NI, CR, PA \\
\hline 16 & & Quercus borucasana Trel. & ME, GU, BE, ES, HO, NI, CR, PA \\
\hline 17 & & Quercus calophylla Schltdl. \& Cham. & $\mathrm{ME}, \mathrm{GU}$ \\
\hline 18 & & Quercus cortesii Liebm.* & ME, GU, BE, HO, NI, CR, PA \\
\hline 19 & & Quercus crassifolia Bonpl. & $\mathrm{ME}, \mathrm{GU}$ \\
\hline 20 & & Quercus crispifolia Trel. & $\mathrm{ME}, \mathrm{GU}, \mathrm{BE}, \mathrm{HO}$ \\
\hline 21 & & Quercus crispipilis Trel. & $\mathrm{ME}, \mathrm{GU}$ \\
\hline 22 & & Quercus elliptica Née & $\mathrm{ME}, \mathrm{GU}, \mathrm{ES}, \mathrm{HO}$ \\
\hline 23 & & Quercus flagellifera Trel. & ME, GU \\
\hline 24 & & Quercus gulielmi-treleasei C.H. Mull.* & $\mathrm{GU}, \mathrm{HO}, \mathrm{CR}, \mathrm{PA}$ \\
\hline 25 & & Quercus salicifolia Née & ME, GU, ES, HO, NI, CR, PA \\
\hline 26 & & Quercus sapotifolia Liebm. & ME, GU, BE, ES, HO, NI, CR, PA \\
\hline 27 & & Quercus skinneri Benth. & ME, GU, ES, HO \\
\hline 28 & & Quercus tristis Liebm. & ME, GU \\
\hline 29 & & Quercus xalapensis Bonpl.* & $\mathrm{ME}, \mathrm{GU}$ \\
\hline
\end{tabular}

*= Especies que representan nuevos registros para el país. 


\section{Resultados}

Actualización de listado de encinos para Guatemala

Se obtuvieron 1,315 registros de Quercus, los cuales corresponden a 29 especies, 12 de la sección Quercus s.s. (encinos blancos) y 17 a la sección $L o-$ batae (encinos rojos) (Tabla 1) (Valencia-A, Coombes $\&$ Villaseñor, 2018). De las especies de encino citadas por Standley y Steyermark (1952) se citan cuatro especies más Quercus cortesii Liebm., Quercus gulielmi-treleasei C.H. Mull. Quercus vicentensis Trel., y Quercus xalapensis Bonpl. (Tabla 1). Alrededor del $50 \%$ de los registros corresponden solamente a cinco especies, siendo estas $Q$. sapotifolia Liebm (13\%), $Q$. peduncularis Née. (13\%), Q. crassifolia Bonpl. (12\%), Q. skinneri Benth. (7\%) y Q. acutifolia Née. (6\%). En contraste, cinco especies presentan menos de diez registros a nivel nacional, las cuales son $Q$. pacayana C.H. Mull., $Q$. polymorpha Schltdl. \& Cham., $Q$. vicentensis Trel., Q. aff. salicifolia Née y Q. xalapensis Bonpl. (Tabla 2). Los departamentos mejor representados en las colectas son: Guatemala (183), Zacapa (155), Huehuetenango (134) y Baja Verapaz (115), mientras que los departamentos con menos de diez registros son $\mathrm{Su}-$ chitepéquez, Retalhuleu y Escuintla. El departamento con mayor riqueza de especies es Zacapa (21), seguido de Huehuetenango (20), Alta Verapaz, Baja Verapaz y Chiquimula (18), mientras los que presentan la menor riqueza son Petén (5), Izabal (3), Suchitepéquez y Escuintla (2) y Retalhuleu con una especie documentada (Tabla 2).

\section{Distribución potencial de las especies de encino a nivel nacional}

Las especies $Q$. sapotifolia, Q. crassifolia, $Q$. lancifolia Schltdl. \& Cham., Q. elliptica y Q. bumelioides Liebm. presentan una amplia distribución en el territorio nacional, dado que registran ejemplares en un amplio rango de altitud y localidades (Tabla 2 y 3 ). Las especies con una distribución más restringida son $Q$. aff. salicifolia, $Q$. vicentensis, $Q$. xalapensis, $Q$. cortesii, y $Q$. polymorpha las cuales su distribución se restringe a zonas más húmedas a lo largo de la cadena montañosa central del país. El resto de especies de encino (19) presentan una amplia distribución (Figura 1 y 2), a lo largo del sistema montañoso del país, principalmente en la cadena volcánica, y las serranías del norte del país.
La distribución potencial para el género Quercus en Guatemala es aproximadamente el $95 \%$ de territorio $(10,663,930 \mathrm{ha})$, siendo el sistema montañoso central donde existe un mayor traslape de la distribución potencial de las especies, y según el modelo generado son hasta 26 especies las que puedan confluir en una misma zona (Figura 3a). Sin embargo, para el 2012 se estimaba que la cobertura forestal ocupaba apenas el 34\% (3,711,366 ha) del territorio (Grupo Interinstitucional de Monitoreo de Bosques y Uso de la Tierra, 2014); del cual, el $91 \%$ corresponde a la distribución potencial de Quercus $(3,382,827 \mathrm{ha})$, restringiendo la probabilidad de ocurrencia a los remanentes boscosos del país (Figura 3b). De esta distribución potencial, el Sigap alberga el 49\% (1,657,585 ha) del área de distribución estimada (Figura 3c), mientras que el resto del área $(1,725,241 \mathrm{ha})$ se encuentra fuera del mismo (Figura 3d). La densidad del área de colecta de encinos, según los registros de herbario, corresponde aproximadamente el 50\% del área estimada para la distribución del género en Guatemala (Figura 4a). El mayor esfuerzo de colecta se registra en la región central, en la cadena volcánica y nororiente del país, principalmente en Sierra de la Minas. Por lo cual, aún existen localidades que no han sido exploradas aún, con alta probabilidad de ocurrencia de especies del género, tal es el caso de los departamentos de Quiché y San Marcos, así como los departamentos en la vertiente del Pacífico de Guatemala (Figura 4b).

\section{Estado de conservación de las especies de encino}

De las 29 especies registradas, según la propuesta para la categorización de LEA seis se ubicaron en la categoría En Peligro Crítico, 22 dentro de la categoría de Amenazada y una en la categoría de Vulnerable (Tabla 3). Por otro lado, 26 especies se encuentran en Lista Roja de Especies Amenazadas de la UICN, una se ubica en la categoría En Peligro, siete en la categoría Vulnerable, siete en la categoría Casi Amenazado, ocho se encuentran como Preocupación Menor y tres especies como Datos insuficientes (Tabla 4).

\section{Discusión}

\section{Actualización del listado de encinos para Gua- temala}

El presente estudio cita cuatro nuevas especies de encinos para el país (Tabla 1), considerando que la última revisión de Quercus fue realizada por Standley 


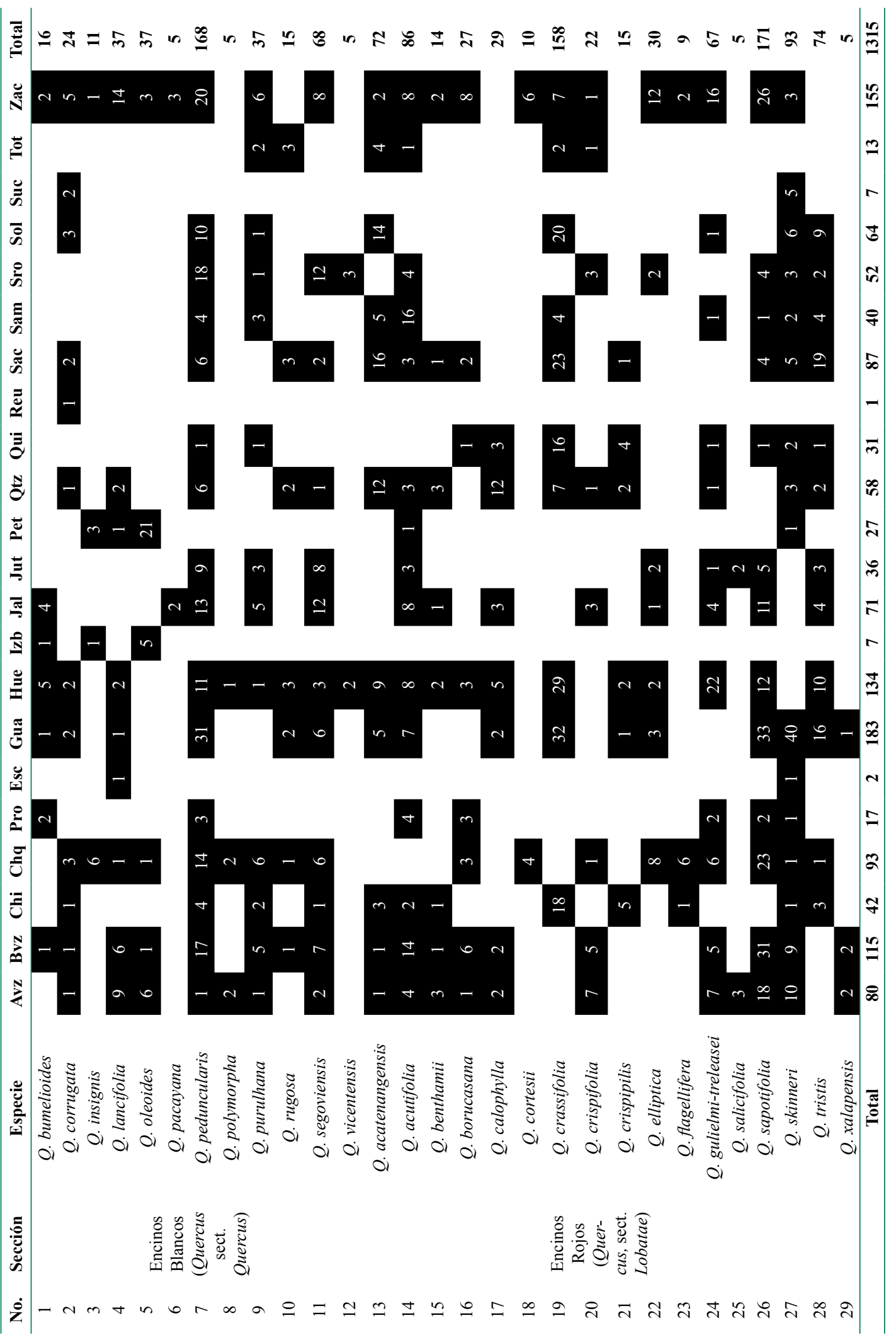




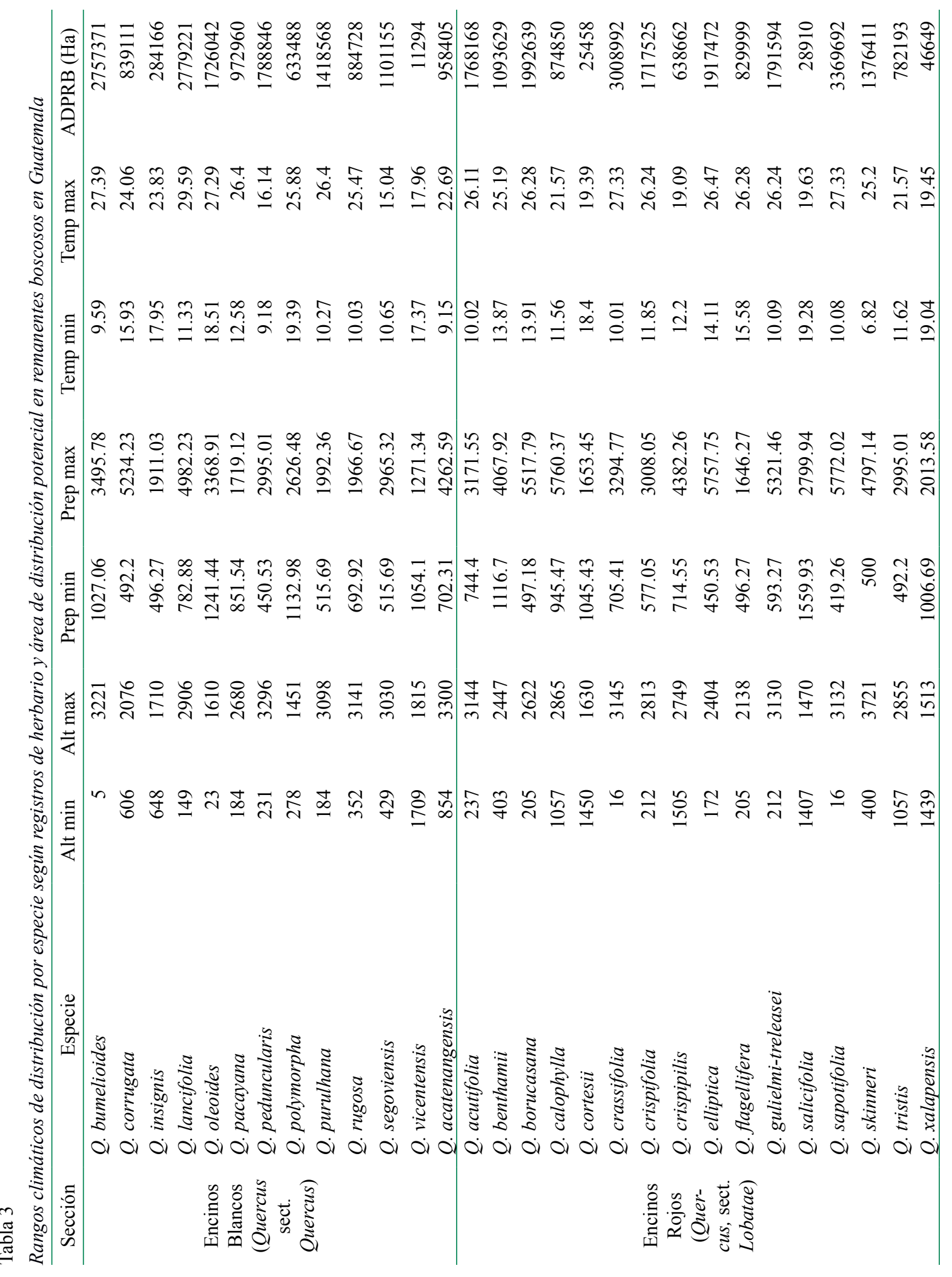




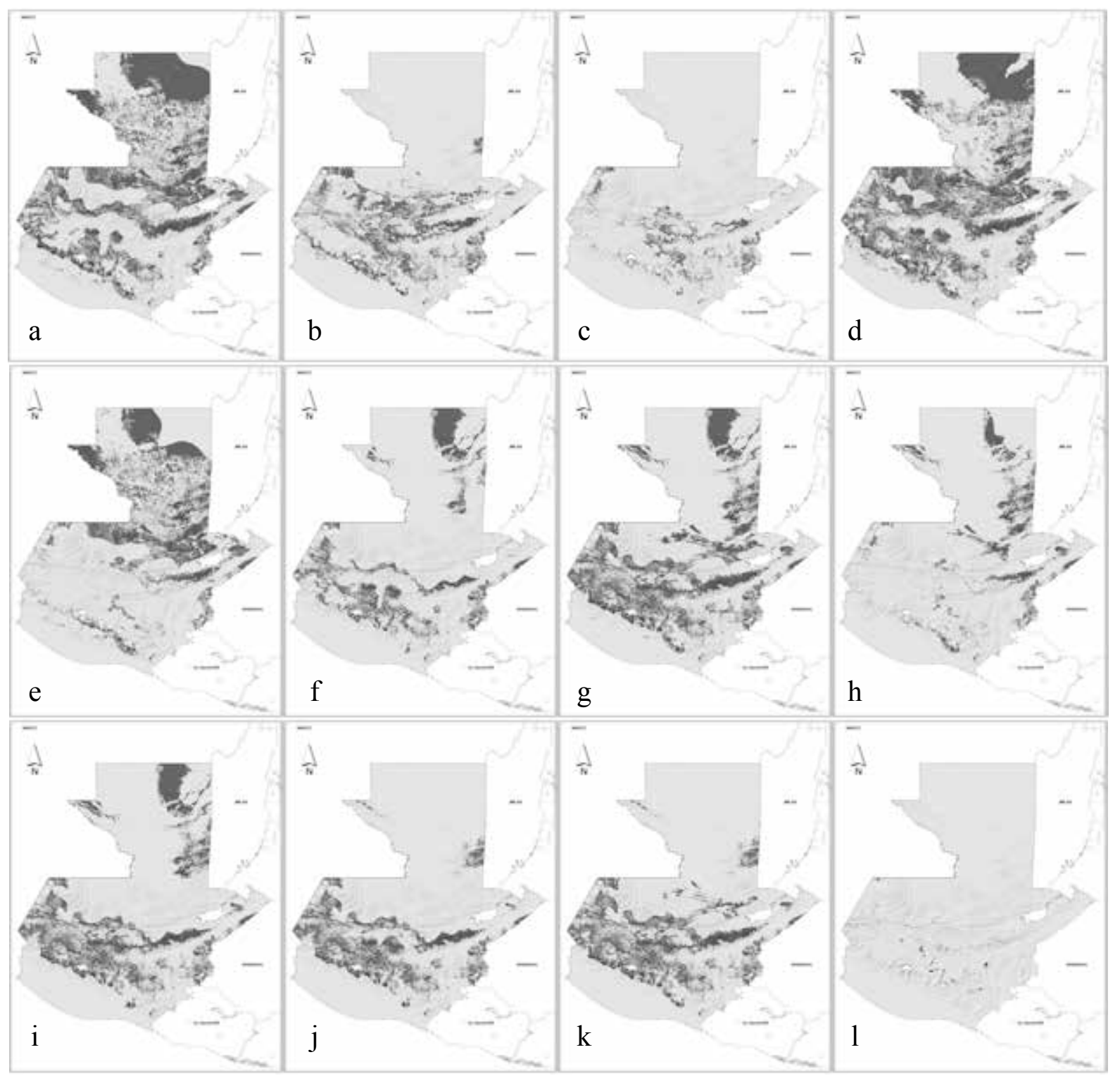

Figura 1. Distribución potencial de las especies de encino blanco (sección Quercus) a. Q. bumelioides, b. Q. corrugata, c. Q. insignis, d. Q. lancifolia, e. Q. oleoides, f. Q. pacayana, g. Q. peduncularis, h. Q. polymorpha, i. Q. purulhana j. Q. rugosa, k. $Q$. segoviensis, 1 . $Q$. vicentensis. 


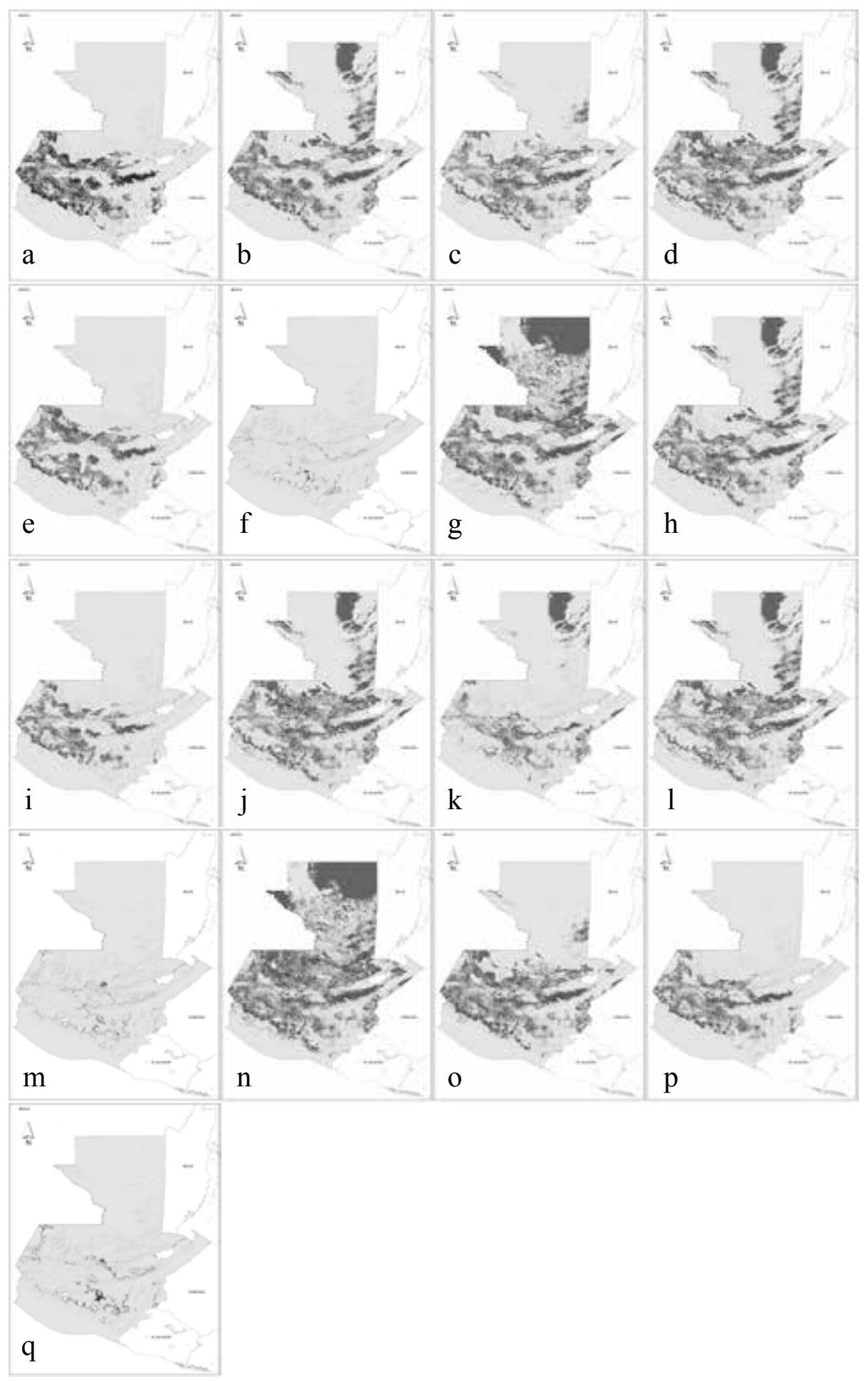

Figura 2. Distribución potencial de las especies de encino rojo (sección Lobatae): a. Q. acatenangensis b. Q. acutifolia, c. $Q$. benthamii, d. Q. borucasana, e. Q. candicans, f. Q. cortesii, g. Q. crassifolia, h. Q. crispifolia, i. Q. crispipilis, j. Q. elliptica, k. Q. flagellifera, 1. Q. gulielmi-treleasei, m. Q. salicifolia, n. Q. sapotifolia, o. Q. skinneri, p. Q. tristis, q. Q. xalapensis. 

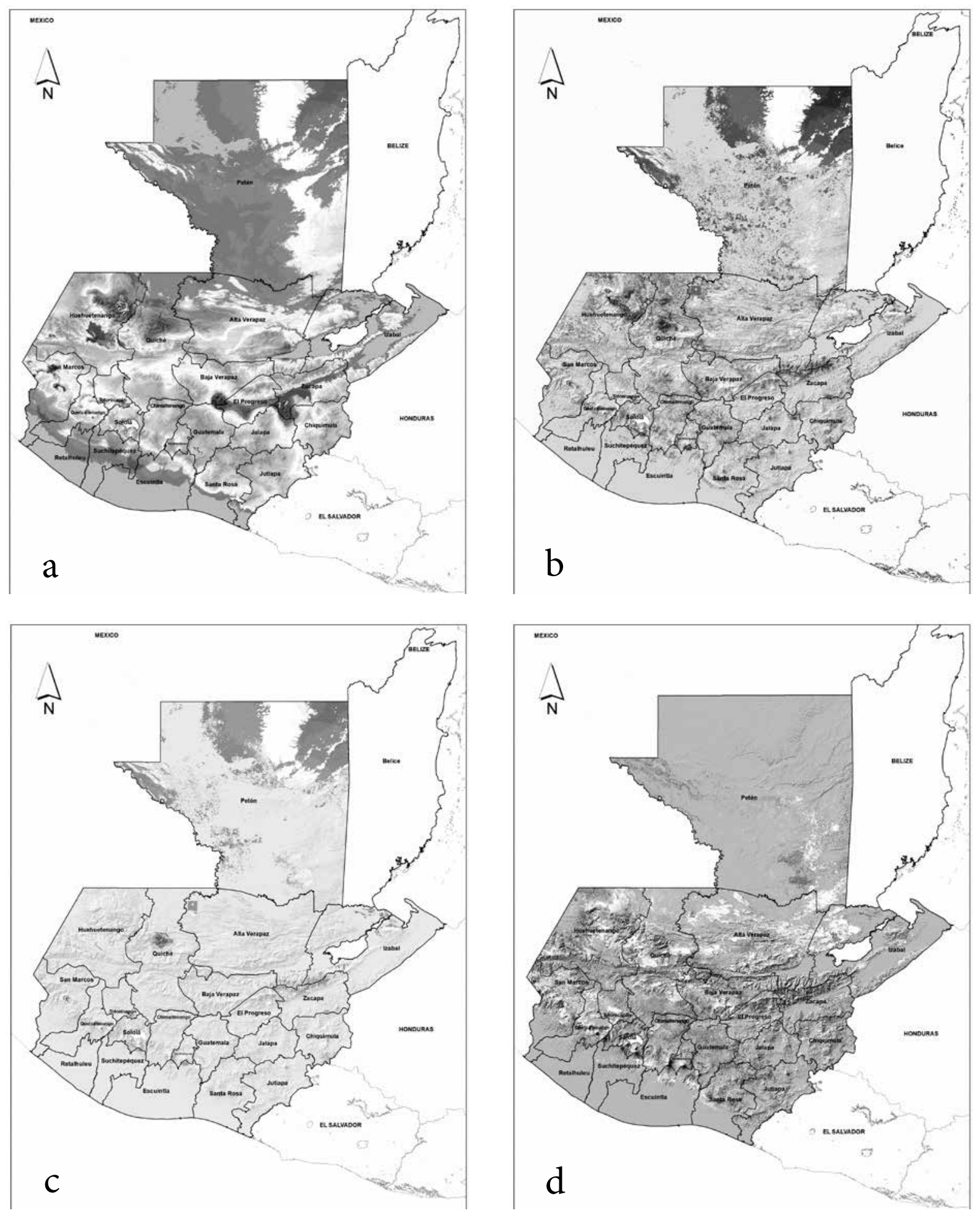

Figura 3. Distribución potencial de las especies de encino: a. Distribución potencial en territorio nacional b. Distribución potencial en áreas con cobertura boscosa (2012). c. Distribución potencial dentro del Sistema Nacional de Áreas Protegidas d. Distribución potencial. en áreas boscosas fuera de área protegidas. 

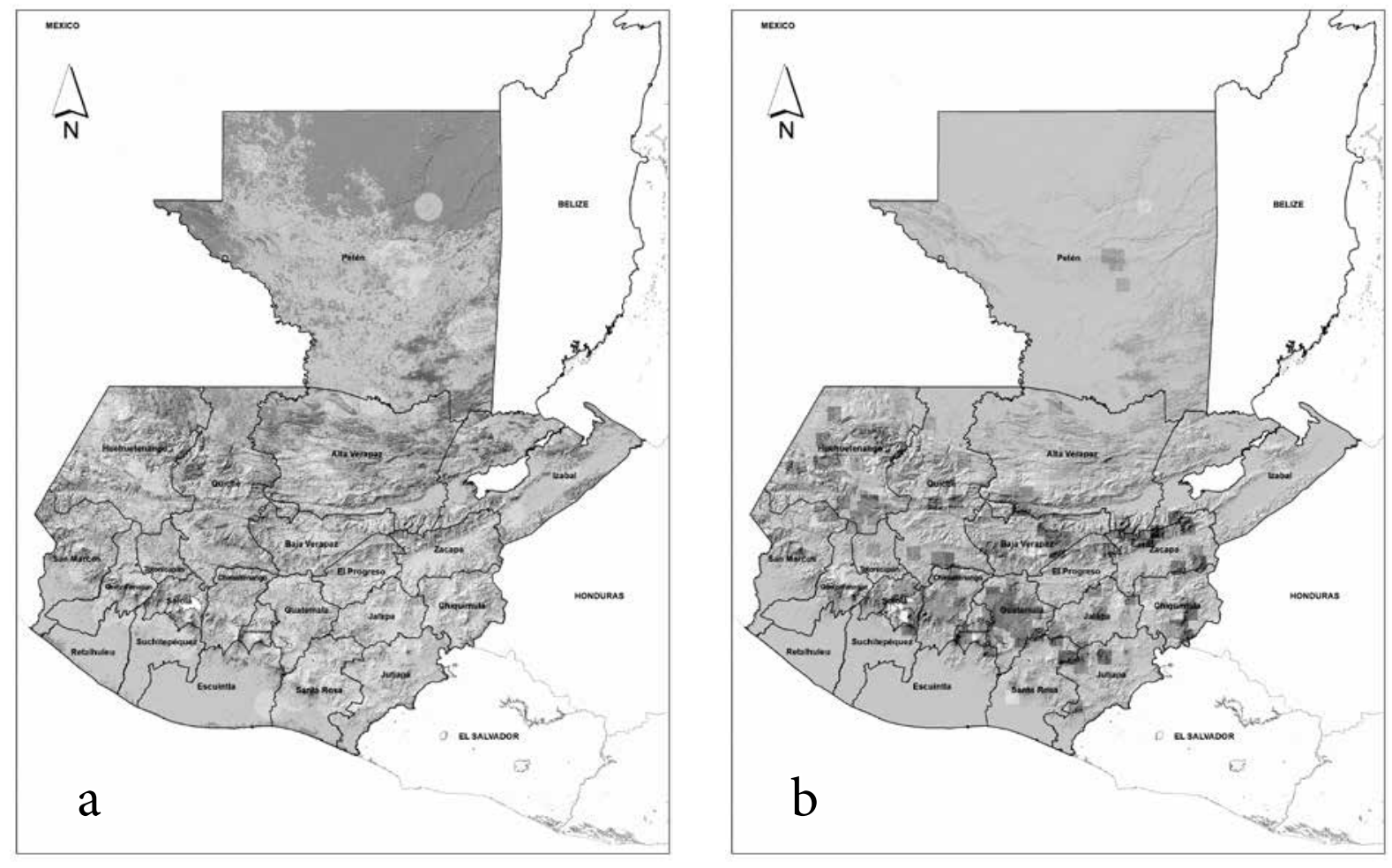

Figura 4. Análisis de vacíos de muestreo: a. Densidad de puntos de colecta en base a la distribución potencial de encinos. b. Distribución de puntos de colecta y coloreados según la probabilidad de ocurrencia de otras especies.

y Steyermark (1952) para la Flora de Guatemala y algunas especies son ahora sinonimias de otras; se resalta la importancia del esfuerzo para comprender este grupo de especies clave en los bosques guatemaltecos. De los nuevos registros, $Q$. xalapensis no había sido citado en la Flora de Guatemala, aunque sí es mencionado por Stevens, y colaboradores. (2001) en la Flora de Nicaragua, mientras que $Q$. cortesii está citado para México, por lo que su distribución más al norte estaba documentada pero no está registrado en Guatemala y Q. gulielmi-treleasei amplían su distribución al norte, esté último se registraba para Costa Rica y Panamá (Jerome, 2017; Nixon, 1998; Vivero, et al, 2006; Wenzell \& Kenny, 2015). Por otro lado, $Q$. vicentensis se registraba para el sur de México y El Salvador, pero no existían colectas en Guatemala. Asimismo, se eviden- cia la alta riqueza de encinos rojos (sección Lobatae) con 17 especies, cinco más que los encinos blancos (sección Quercus). Cabe resaltar, que los encinos rojos se encuentran asociados principalmente a bosques con mayor humedad como los bosques de niebla y selvas lluviosas. Asimismo, Torres-Miranda y colaboradores (2014) y Rodríguez-Correa (2015) resaltan la importancia de Mesoamérica como un punto importante de endemismo de encinos rojos. Por otro lado, los encinos blancos se asocian a ecosistemas más secos y donde tienden ser las especies dominantes (González-Rivera, 1993; Nixon, 1993; Torres-Miranda, 2014; Torres-Miranda, Luna-Vega y Oyama, 2011; Valencia-A., 2004). Por tanto, se hace notorio la importancia del género en el desarrollo de los diferentes bosques que conforman los diferentes ecosistemas del país. 
Tabla 4

Categorías de Amenaza de las especies de encino para Guatemala según UICN y la propuesta según los criterios de LEA (2017) para la nueva re-categorización de la flora nacional

\begin{tabular}{|c|c|c|c|}
\hline Sección & Nombre Aceptado & Evaluación UICN & $\begin{array}{l}\text { Categoría según } \\
\text { propuesta } 2017\end{array}$ \\
\hline \multirow{12}{*}{$\begin{array}{l}\text { Quercus s.s., } \\
\text { Subgenus } \\
\text { Lepidobalanus } \\
\text { (Encinos } \\
\text { blancos) }\end{array}$} & Bumelioides & NT & Amenazada \\
\hline & Corrugata & No evaluado & Amenazada \\
\hline & Insignis & NT & En Peligro Crítico \\
\hline & Lancifolia & NT & Amenazada \\
\hline & Oleoides & VU & Amenazada \\
\hline & Pacayana & NT & Amenazada \\
\hline & Peduncularis & $\mathrm{LC}$ & Amenazada \\
\hline & Polymorpha & VU & En Peligro Crítico \\
\hline & Purulhana & VU & Amenazada \\
\hline & Rugosa & $\mathrm{LC}$ & Amenazada \\
\hline & Segoviensis & $\mathrm{DD}$ & Amenazada \\
\hline & Vicentensis & VU & En Peligro Crítico \\
\hline \multirow{17}{*}{$\begin{array}{l}\text { Lobatae, } \\
\text { Subgenus } \\
\text { Erythro- } \\
\text { balanus (En- } \\
\text { cinos negros o } \\
\text { rojos) }\end{array}$} & Acatenangensis & $\mathrm{LC}$ & Amenazada \\
\hline & Acutifolia & $\mathrm{LC}$ & Amenazada \\
\hline & Benthamii & VU & Amenazada \\
\hline & Borucasana & No evaluado & Amenazada \\
\hline & Calophylla & $\mathrm{LC}$ & Amenazada \\
\hline & Cortesii & DD & En Peligro Crítico \\
\hline & Crassifolia & $\mathrm{LC}$ & Amenazada \\
\hline & Crispifolia & NT & Amenazada \\
\hline & Crispipilis & NT & Amenazada \\
\hline & Elliptica & $\mathrm{EN}$ & Amenazada \\
\hline & Flagellifera & VU & Amenazada \\
\hline & Gulielmi-treleasei & $\mathrm{DD}$ & Amenazada \\
\hline & Salicifolia & No evaluado & En Peligro Crítico \\
\hline & Sapotifolia & $\mathrm{LC}$ & Vulnerable \\
\hline & Skinneri & NT & Amenazada \\
\hline & Tristis & $\mathrm{LC}$ & Amenazada \\
\hline & Xalapensis & VU & En Peligro Crítico \\
\hline
\end{tabular}

EN= En Peligro; VU= Vulnerable; NT= Casi Amenazado; $\mathrm{LC}=$ Preocupación Menor; $\mathrm{DD}=$ Datos insuficientes. 
Distribución potencial de las especies de encino a nivel nacional

Los modelos de distribución de especies han sido utilizados para comprender procesos ecológicos, biogeográficos, y para generar estrategias de conservación de la diversidad biológica; éstos pueden basarse en información de parámetros climáticos, requerimientos de hábitat, estructura del paisaje, entre otros factores (Elith et al., 2006). Los mapas generados para las especies de Quercus representan la probabilidad de ocurrencia según los rangos en los valores de precipitación, temperatura y altitud por especie (Tabla 3 ), siendo una primera aproximación para comprender la distribución de éstas especies en el territorio guatemalteco (Figuras 1-3). Es importante mencionar que existen otros modelos y algoritmos que pueden ser utilizados para generar dichos modelos (Elith et al., 2006), pero a la fecha aún no se cuenta con un consenso sobre cuál de las herramientas propuesta es la más efectiva para predecir la distribución de las especies (Naoki, Gómez, López, Menenses, $\&$ Vargas, 2006). Como todos los modelos, el que se presenta en este estudio tiene sus limitantes (Mateo, Felicísimo, \& Muñoz, 2011), como el no considerar interacciones biológicas y edáficas, así como la historia evolutiva de cada una de las especies. A pesar de ello, este primer esfuerzo nos aproxima a comprender la distribución potencial así como los vacíos de información de este grupo.

El mapa de distribución acumulado de las especies de encino (Figura 4a) identifica las áreas con mayor ocurrencia de las especies, la cual se ubica en la cadena volcánica y las regiones montañosas del país, en donde la probabilidad de una co-ocurrencia de especies es alta (26 especies). Esto se evidencia en sitios de colecta realizados durante el 2015 a 2017, donde se reporta co-ocurrencias entre cinco a siete especies en un área de 0.1 ha (Quezada et al., 2016). Patrones similares, donde los bosques dominados por encino, tienden a presentar una alta riqueza de especies, pueden observarse en México (Encina-Dominguez \& Villareal-Quintanilla, 2002; Luna-Vega, Alcántara-Ayala, Ruíz-Jiménez, \& Contrera Medina, 2006; Zavala-Chavez, 1998), siendo el país de América con el mayor número de especies (161) (Valencia-A, 2004). Es importante destacar que la mayoría de encinos presentan distribuciones restringidas a nivel biogeográfico (Torres-Miranda, 2014) y un alto recambio de especies a nivel latitudinal y altitudinal (Rodríguez-Correa et al., 2017) explicando la probabilidad de co-ocurrencia de estas especies en los modelos de distribución generados. Sin embargo, hay que considerar que la mayoría de especies presentan menos de 50 registros, evidenciando los vacíos de información para el grupo (Tabla 2, Figura 5b), por lo cual, es importante reforzar los esfuerzos de colecta sistemática de este grupo para comprender los requerimientos ecosistémicos que determinan la riqueza y distribución de este género.

La escasa información a nivel nacional de este grupo de especies (Tabla 2, Figura 4b), estima que al menos el 50\% del área de distribución no cuenta con colectas del género (Figura 4a), aunado a que alrededor de la mitad de los registros corresponden solamente a cinco especies, cuyos registros están en la región central y nororiente del país (Tabla 2, Figura 4b). Los principales vacíos se hacen evidentes en la región norte y occidente del país en los departamentos de Petén, Izabal, Alta Verapaz, Quiché y Huehuetenango, así como los departamentos que se encuentran en la vertiente del pacífico, San Marcos, Retalhuleu, Suchitepéquez y Escuintla. La escasa documentación de la diversidad biológica, así como los vacíos de información que se presentan en este grupo coinciden con otros autores (Bermúdez \& Sánchez, 2000; Estrada, Garcia, \& Machuca, 2015; Islebe, Cleef, \& Velázquez, 1994; Morales et al., 2009; Pérez, 2009; Quezada et al. 2016; Sánchez; 2010) donde destacan la importancia de incrementar los esfuerzos para recabar la información biológica sobre este grupo, principalmente en el sistema montañoso del país.

\section{Estado de conservación}

De las especies registradas para el país, 11 presentan distribución en todo el istmo centroamericano, 18 en el bloque de Mesoamérica nuclear y de estos 8 son endémicos de la región que conforma el sur de México y Guatemala (Tabla 1). Es importante resaltar que todas las especies a excepción de Q. rugosa presentan distribución restringida del sur de México a Centroamérica. Por lo cual, pueden considerarse endémicas regionales, resaltando la importancia de este grupo en la historia natural del país. Sin embargo, la mayoría de estas especies presentan un grado de amenaza ya que se ubican en la categoría de Vulnerable según la Lista Roja de Especies Amenazadas de UICN, siendo estas $Q$. oleoides, $Q$. polymorpha, $Q$. purulhana, $Q$. vicentensis, $Q$. benthamii, $Q$. flagellifera y $Q$. xalapensis. Un taxón es Vulnerable cuando la evidencia disponible indica que se está enfrentando a un riesgo alto de extinción 


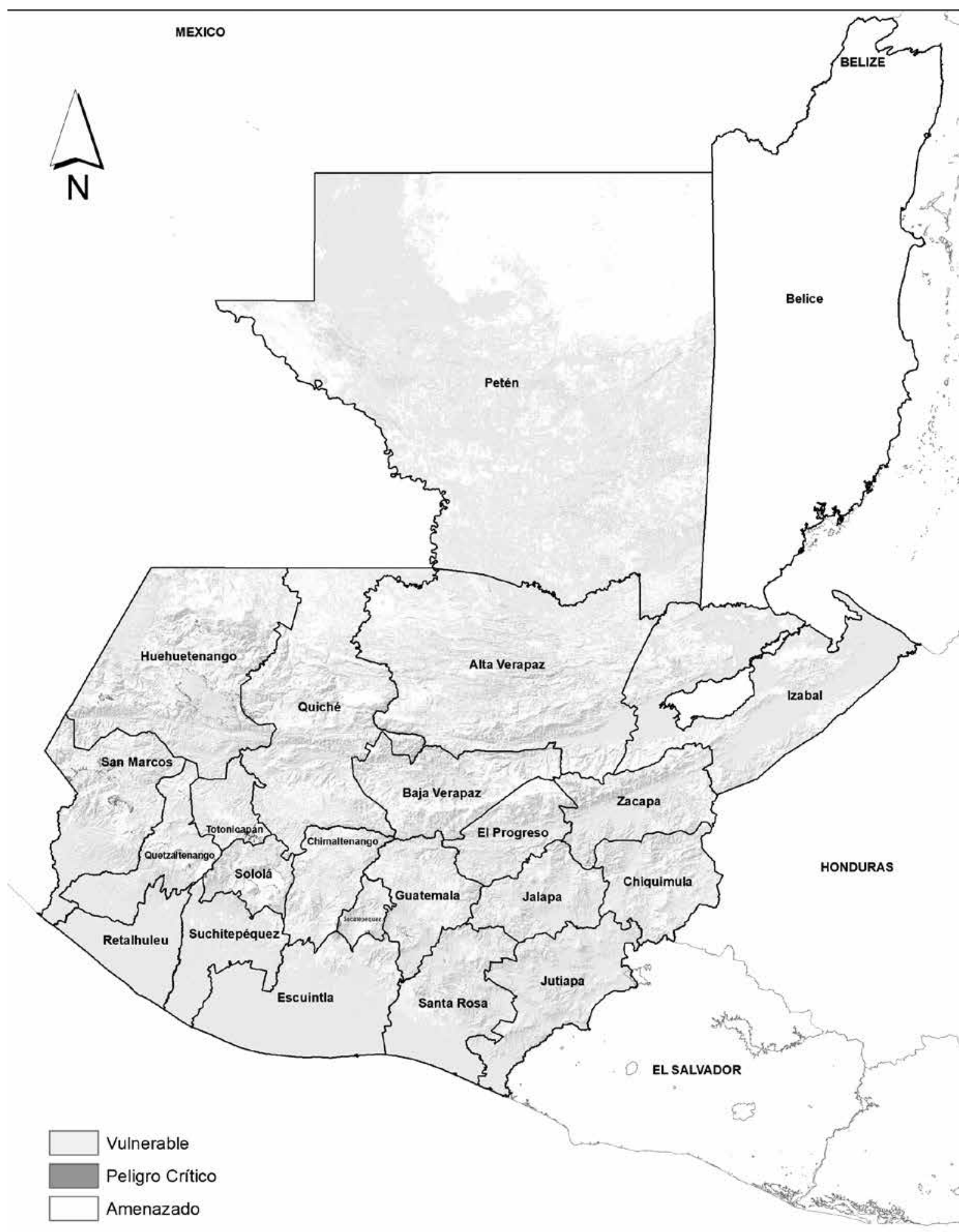

Figura 5. Distribución potencial de las especies de encino según su estado de conservación. 
en estado silvestre (Unión Internacional para la Conservación de la Naturaleza, 2001). La especie Quercus elliptica Née se ubica en la categoría En Peligro, ya que la evidencia disponible indica que se está enfrentando a un riesgo muy alto de extinción en estado silvestre (UICN, 2001). Las especies $Q$. insignis, $Q$. polymorpha, $Q$. vicentensis, $Q$. cortesii, $Q$. aff. salicifolia y $Q$. xalapensis, las cuales presentaron un mayor grado de amenaza (Tabla 4), son a su vez las que presentan menos registros en las colecciones de herbario. Esta situación se debe probablemente a que no son las especies más abundantes y por las similitudes con otras especies, se hace difícil diferenciarlas en campo, por lo que pudieron haber pasado desapercibidas por los colectores. Asimismo, se debe resaltar que la mayoría de especies (28) está dentro de alguna categorización (Tabla 4), las cuales cuentan con poca información acerca de los requerimientos de hábitat (Rodríguez-Correa et al., 2015, 2017; Torres-Miranda, 2014). Esto debe conducir a la generación de una alerta para incrementar los esfuerzos para su estudio y con ello establecer estrategias de manejo y conservación efectivas para asegurar su permanencia, más aún si se considera que su distribución está restringida al istmo centroamericano (Muller, 1942; Standley \& Steyermark, 1942; Torres-Miranda, 2014), donde la deforestación y cambio de uso de suelo son las principales amenazas de la diversidad biológica (Aide et al., 2012; Quezada et al., 2014). Sin embargo, se debe resaltar que el sistema guatemalteco de áreas protegidas juega un papel crucial para la conservación de las especies de encino, así como los remanentes boscosos fuera del sistema. Por lo cual, la conectividad de estos remanentes es fundamental para el mantenimiento de los flujos y procesos ecológicos que permiten los bienes y servicios que brindan esta especies a la población guatemalteca.

\section{Agradecimientos}

La realización de este trabajo, ha sido posible gracias al cofinanciamiento de Digi-Usac-2015-2017 a los Proyectos (4.8.63.7.40-2015; 4.8.63.4.05-2016; 4.8.63.4.01-2017). Asimismo, agradecemos al personal de los Herbarios Agua de la Facultad de Agronomía, Bigu de la Escuela de Biología, Uval de la Universidad del Valle por facilitar el acceso y revisión de los especímenes de encinos presentes en la colección de referencia. A los revisores de este trabajo por los aportes y recomendaciones al mismo.

\section{Referencias}

ArcGis (versión 10.3) [Software de computación]. California: Esri.

Aide, T. M., Clark, M. L., Grau, H. R., López-Carr, D., Levy, M. A., Redo, D., ... Muñiz, M. (2012). Deforestation and reforestation of Latin America and the Caribbean (2001-2010). Biotropica, 45, 262-271. doi: 10.1111/j.1744-7429.2012.00908.x Alianza para la Conservación de los Bosques de Pino-Encino de Mesoamérica. (2008). Plan de Conservación de los Bosques de Pino-Encino de Centroamérica y el Ave Migratoria Dendroica chrysoparia. Guatemala: Fundación Defensores de la Naturaleza y The Nature Conservacy. Recuperado de http://www.pronatura-sur.org/web/ docs/PLAN_CONSERVACION_bosques_pino-encino.pdf

Balick, M. J., Nee, M. H., \& Atha, D. E. (2000). Checklist of the vascular plants of Belize, with common names and uses. Bronx, New York: New York Botanical Garden Press.

Bermúdez, M., \& Sánchez, G. J. (2000). Identificación de vacios de información botánica en Centroamérica. San José, Costa Rica: WWF Centroamérica, Museo Nacional de Costa Rica y Red de Herbarios de Mesoamérica y el Caribe.

Consejo Nacional de Áreas Protegidas. (2009). Lista de especies amenazadas de Guatemala. Guatemala: Autor.

Convention on Biological Diversity. (2010). Report of the tenth meeting of the conference of the parties to the convention on biological diversity. En tenth meeting of the Conference of the Parties to the Convention on Biological Diversity, Nagoya, Japan.

Elith, J., Graham, C. H., Anderson, R. P., Dudık, M., Ferrier, S., Guisan, A., ... Zimmermann, N. E. (2006). Novel methods improve prediction of species' distributions from occurrence data. Ecography, 29, 129-151.

Encina Domínguez, J. A., \& Villarreal Quintanilla, J. A. (2002). Distribución y aspectos ecológicos del género Quercus (Fagaceae), en el estado de Coahuila, México. Polibotánica, (13), 1-23. 
Estrada, C., García, M., \& Machuca, O. (2015) Análisis de la efectividad ecológica de los espacios terrestres incluidos en el Sistema Guatemalteco de Áreas Protegidas (SIGAP). Ciencia y Conservación, 6, 13-13.

Flores Arzú, R., Comandini, O., \& Rinaldi, A. C. (2012). A preliminary checklist of macrofungi of Guatemala, with notes on edibility and traditional knowledge. Mycosphere, 3, 1-21. doi: 10.5943/ mycosphere/3/1/1

Fondo Nacional para la Conservación/Consejo Nacional de Áreas Protegidas (en prensa) Informe Final Proyecto F14/2016/FONACON: Actualización de la Lista de Especies Amenazadas (LEA) de Guatemala.

Gall, F. (1976). Diccionario Geográfico de Guatemala. Instituto Geográfico Nacional "Ingeniero Alfredo Obiols Gómez”. Guatemala: Instituto Geográfico Nacional.

García, B. L. (1998). Estudio del dosel de la selva nublada del biotopo universitario para la conservación del quetzal, Lic. Mario Dary Rivera (Tesis de licenciatura). Universidad de San Carlos de Guatemala, Guatemala, Facultad de Ciencias Químicas y Farmacia, Guatemala.

González-Rivera, R. (1993). La diversidad de encinos mexicanos. Boletín de la Sociedad Botánica de México, 44, 125-142.

Grupo Interinstitucional de Monitoreo de Bosques y Uso de la Tierra. (2014). Mapa de bosques y uso de la tierra 2012 y Mapa de cambios en uso de la tierra 2001- 2010 para estimación de emisiones de gases de efecto invernadero. Documento Informativo. Guatemala: Autor.

Islebe, G. A., Cleef, A. M., \& Velázquez, A. (1994). Especies leñosas de la sierra de los Cuchumatanes y de la cadena volcánica, Guatemala. Acta Botánica Mexicana, (29), 83-92.

Jerome, D. (2017). Quercus polymorpha. The IUCN Red List of Threatened Species 2017: e. T194221A2304665.

Kappelle, M. (2006). Structure and composition of Costa Rican montane oak forests. En M. Kappelle (Ed.), Ecology and conservation of neotropical montane oak forests (pp. 127-139). Berlin: Springer.
Luna-Vega, I., Alcántara-Ayala O., Ruíz-Jiménez, C. A., \& Contreras-Medina, R. (2006) Composition and structure of humid montane oak forests at different sites in Central and Eastern Mexico. En M. Kappelle (Ed.). Ecology and conservation of neotropical montane oak forests (pp. 3-13). Berlin: Springer.

Manos, P. S., Doyle, J. J., \& Nixon, K. C. (1999). Phylogeny, biogeography, and processes of molecular differentiation in Quercus subgenus Quercus (Fagaceae). Molecular Phylogenetic and Evolution, 12(3), 333-349. doi: 10.1006/mpev.1999.0614

Marañón, T., Padilla Díaz, C. M., Pérez Ramos, I. M., \& Villar, R. (2014). Tendencias en la investigación sobre ecología y gestión de las especies de Quercus. Ecosistemas, 23(2), 124-129. doi: 10.7818/ ECOS.2014.23-2.16.

Mateo, R. G., Felicísimo, A. M., \& Muñoz, J. (2011). Modelos de distribución de especies: Una revisión sintética. Revista Chilena de Historia Natural, 84(2), 217-240.

Melgar, W. (2003). Estado de la diversidad biológica de los árboles y bosques de Guatemala. Manuscrito inédito. En Documentos de Trabajo sobre Recursos Genéticos Forestales (Servicio de Desarrollo de Recursos Forestales, Documento de Trabajo FGR/53S). Roma: Italia: Organización de las Naciones Unidas para la Agricultura y Alimentación.

Morales, O., García, E., Cáceres, R., Bran, M. C., Gurriarán, N., \& Flores, R. (2009). Gasteromycetes de Guatemala: especies citadas en el período de 1948 a 2008. Revista cientifica Instituto de Investigaciones Químicas y Biológicas, 5(1), 27-33.

Moreno, G. B. (2006). Economic Valuation of water supply as a key environmental service provided by Montane Oak Forest watershed areas in Costa Rica. En M. Kappelle (Ed.), Ecology and conservation of neotropical montane oak forests (pp. 435-446). Berlin: Springer.

Muller, C. H. (1942). The Central American species of Quercus (Missellaneous, No. 477). Washington, D.C.: Departament of Agricultura, Government Printing Office.

Naoki, K., Gómez, M. I., López, R. P., Meneses, R. I., \& Vargas, J. (2006). Comparación de modelos de distribución de especies para predecir la dis- 
tribución potencial de vida silvestre en Bolivia. Ecología en Bolivia, 41(1), 65-78.

Nixon, K.C. (1993). The genus Quercus in Mexico. En T.P. Ramammoorthy, R. Bye, A. Lot \& J. Fa (Eds.). Biological diversity of Mexico: Origins and distribution (pp. 447-458). Nueva York: Oxford University Press.

Nixon, K. (1998). Quercus benthamii. The IUCN Red List of Threatened Species, e.T35961A9970290. doi: $10.2305 /$ IUCN.UK.1998.RLTS. T35961A9970290.en

Nixon, K. (1998b). Quercus bumelioides. The IUCN Red List of Threatened Species, e. T32760A9727972. doi: 10.2305/IUCN.UK.1998. RLTS.T32760A9727972.en.

Nixon, K. (1998c). Quercus flagellifera. The IUCN Red List of Threatened Species, e. T35965A9970562. doi: 10.2305/IUCN.UK.1998. RLTS.T35965A9970562.en.

Nixon, K. (1998d). Quercus gulielmitreleasei. The IUCN Red List of Threatened Species, e. T35966A9970641. doi: 10.2305/IUCN.UK.1998. RLTS.T35966A9970641.en.

Nixon, K. (1998e). Quercus purulhana. The IUCN Red List of Threatened Species, e. T32766A9723016. doi: 10.2305/IUCN.UK.1998. RLTS.T32766A9723016.en.

Nixon, K. (1998f). Quercus vicentensis. The IUCN Red List of Threatened Species, e. T34685A9878246. doi:10.2305/IUCN.UK.1998. RLTS.T34685A9878246.en.

Nixon, K. et al. (1998g). Quercus xalapensis. The IUCN Red List of Threatened Species, e. T34686A9878398. doi: 10.2305/IUCN.UK.1998. RLTS.T34686A9878398.en.

Nixon, K. C. (2006). Global and neotropical distribution and diversity of oak (genus Quercus) and oak forests. En M. Kappelle (Ed.). Ecology and conservation of neotropical montane oak forests (pp. 3-13). Berlin: Springer.

Pérez, M. E. (2009). Catálogo de las hepáticas (Marchantiophyta) de Guatemala: una actualización. Brenesia, 71, 3-12.

Pérez-Ramos, I. M., Villar, R., \& Marañón T. (2014). El fascinante mundo de los Quercus: Desde la biología molecular hasta la ecología de las comu- nidades. Ecosistemas, 23(2), 1- 4. doi: 10.7818/ ECOS.2014.23-2.01.

Quezada, M. L., Arroyo-Rodríguez, V., Pérez-Silva, E., \& Aide, T. M. (2014). Land cover changes in the Lachúa región, Guatemala: patterns, proximate cause, and underlying forces over the last 50 year. Regional Environmental Change, 14(3), 1139-1149.

Quezada, M. L., Rodas-Duarte, L., \& Marroquín-Tintí, A. (2016a). Diversidad de encinos de Guatemala, una alternativa para bosques energéticos, seguridad alimentaria y mitigación al cambio climático, Fase I: Las Verapaces y Petén. (INF-2015-18). Guatemala: Universidad de San Carlos de Guatemala, Dirección General de Investigación y Facultad de Ciencias Químicas y Farmacia.

Quezada, M. L., Rodas-Duarte, L., \& Marroquín-Tintí, A. (2016b). Diversidad de encinos de Guatemala, una alternativa para bosques energéticos, seguridad alimentaria y mitigación al cambio climático, Fase I: Jutiapa, Jalapa y Santa Rosa. (INF-201637). Guatemala: Universidad de San Carlos de Guatemala, Dirección General de Investigación y Facultad de Ciencias Químicas y Farmacia.

Rodríguez-Correa, H. A. (2015). Patrones de distribución y filogeografía de los encinos (Quercus: Fagaceae) en Mesoamérica y los Andes (Tesis de doctorado). Universidad Nacional Autónoma de México, México.

Rodríguez-Correa, H., Oyama, K., MacGregor-Fors, I., \& González-Rodríguez, A. (2015). How are oaks distributed in the neotropics? A perspective from species turnover, areas of endemism, and climatic niches. International Journal of Plant Sciences, 176(3), 222-231. doi: 10.1086/679904

Rodríguez-Correa, H., Oyama, K., Quesada, M., Fuchs, E. J., Quezada, M., Ferrugino, L., ... González-Rodríguez, A. (2017). Complex phylogeographic patterns indicate Central American origin of two widespread Mesoamerican Quercus (Fagaceae) species. Tree Genetics \& Genomes, 13(62) doi: 10.1007/s11295-017-1147-7

Rzedowski, J. (1978). Vegetación de México. México, D.F.: Limusa.

Sánchez, D. A. (2010). Identificación de vacios de conservación y priorización de un portafolio de áreas 
protegidas potenciales en bosques de montaña de Guatemala utilizando a las lagartijas arborícolas del género Abronia (Sauria: Anguidae) como modelo. (Tesis de Máster). Universidad Autónoma de Madrid, Madrid, España.

Standley, P. C., \& Steyermark, J. A. (1952). Flora of Guatemala. Fieldiana: Botany, 24(3), 369-396.

Stevens, W. D., Ulloa, C., Pool, A., \& Montiel, O. M. (2001). Flora de Nicaragua. Angiospermas (Fabaceae-Oxalidaceae) (Vol. 85, Tomo 2). Missouri: Botanical Garden Press.

Torres-Miranda, C. A. (2014). Importancia de los ensambles de especies y de los procesos geológico-climáticos en la comprensión de los mecanismos de evolución de la sección Lobatae del género Quercus en América del Norte (Tesis de doctorado). Universidad Autónoma de México, Morelia, Michoacán, México.

Torres-Miranda, C. A., Luna-Vega, I., \& Oyama, K. (2011). Conservation biogeography of red oaks (Quercus, section Lobatae) in Mexico and Central America. American Journal of Botany, 98(2), 290-305.

Unión Internacional para la Conservación de la Naturaleza. (2001). Categorias y Criterios de la Lista Roja de la UICN: Versión 3.1. Gland, Suiza y Cambridge, Reino Unido: UICN.
Valencia-A, S., (2004). Diversidad del género Quercus (Fagaceae) en México. Boletín de la Sociedad Botánica de México, 75, 33-53.

Valencia-A, S., Flores-Franco, G., \& Jiménez-Ramírez, J. (2015). A nomenclatural revision of Quercus acutifolia, Q. conspersa and Q. grahamii (Lobatae, Fagaceae). Phytotaxa, 218(3), 289-294.

Valencia-A, S., Coombes, A. \& Villaseñor, J. (2018). Quercus candicans (Fagaceae) is not a Quercus but a Roldana (Asteraceae). Phytotaxa, 333(2), 251-258.

Vivero, J., Szejner, M., Gordon, J., \& Magin, G. (2006). The red list of trees of Guatemala. Cambridge: Fauna \& Flora International.

Wenzell , K. \& Kenny, L. (2015). Quercus acutifolia. The IUCN Red List of Threatened Species, e.T78803712A78803830. doi:10.2305/IUCN. UK.2015-4.RLTS.T78803712A78803830.en.

Wenzell , K. \& Kenny, L. (2015B). Quercus crassifolia. The IUCN Red List of Threatened Species, e.T194090A2297643. doi:10.2305/IUCN. UK.2015-4.RLTS.T194090A2297643.en.

Zavala-Chávez, F. (1998). Observaciones sobre la distribución de encinos en México. Polibotánica, $8,47-64$. 